\title{
Inclusion of Frequency Dependent Parameters in Power Transmission Lines Simulation using Harmonic Analysis and Proper Generalized Decomposition
}

\author{
M.H. Malik ${ }^{\mathrm{a}}$, D. Borzacchiello ${ }^{\mathrm{a}, *}$, F.Chinesta $^{\mathrm{a}}$, P.Diez $^{\mathrm{b}}$ \\ ${ }^{a}$ Institut de Calcul Intensif (ICI), École Centrale de Nantes, 1 rue de la Noë, BP 92101, \\ 44321 Nantes cedex 3, France \\ ${ }^{b}$ Laboratori de càlcul numèric (LaCàN), C2 Campus Nord, Polytecnic University of \\ Catalunya, Calle Jordi Girona, 31, 08034 Barcelona, Spain
}

\begin{abstract}
This study presents the application of Proper Generalized Decomposition on transmission line models involving frequency dependent parameters. Frequency dependence of parameters can be due to a number of reasons including phenomena such as "Ground Return Effects", "Proximity Effects" and "Skin Effects". In the current study, simplified methods of skin effects based on Bessel functions are used in order to showcase the method. Although we implement our study using a specific model for skin effects to demonstrate the effectiveness of the proposed method to accomodate frequency dependent effects in PGD, the present work is not meant to discuss the merits of any particular skin effects model. The method can easily accommodate other effects which induces frequency dependence in the transmission line parameters. In time domain modeling, the parameters are assumed constant
\end{abstract}

\footnotetext{
${ }^{*}$ Corresponding author

Email addresses: muhammad.malik@ec-nantes.fr (M.H. Malik), domenico.borzacchiello@ec-nantes.fr (D. Borzacchiello), francisco.chinesta@ec-nantes.fr (F.Chinesta), pedro.diez@upc.edu (P.Diez)
} 
and these models prove inefficient when incorporating these parameters as function of frequency. Therefore, a frequency domain simulation is implemented using harmonic analysis. PGD presents a separated representation and provides a quick and accurate solution for such problems.

Keywords: Skin Effect, Frequency Dependent Parameters, PGD, Transmission Lines, Fractional Derivative

\section{Introduction}

Accurate modeling of transmission lines is important in the simulation and subsequent transient analysis of power grids. In this framework, frequency dependence of the line parameters should be accounted for [1]. The parameters of transmission lines in practice are highly frequency dependent and it is necessary to accommodate the frequency dependence over the whole frequency range of interest.

$\mathrm{AC}$ transmission lines are affected by the frequency of the $\mathrm{AC}$ voltage which produces a secondary effect on the line resistance and inductance. This effect is related to the non uniform distribution of the current which tends to concentrate more towards the outer surface of the line away from the center as the frequency increases [2]. Skin effect results in the reduction of the effective cross-section of the line used for the transmission of current and hence the resistance increases. This concentration of current at the surface has an inverse relation with the internal inductance of the line, as the frequency increases the inductance decreases [3, 4]. There are other phenomena like ground return and proximity which also have an effect on the transmission line parameters resulting in the frequency dependence of these parameters 3 . 
If the phenomena which produce frequency dependence are ignored and the parameters are assumed to be constant, this results in a magnification of the higher harmonics [5]. The wave shapes are distorted and at higher frequency the peaks have higher magnitudes than that are observed in practice. There are a wide range of frequencies present in the signals during the transient phase and this can be modeled with the frequency-dependent parameters.

The challenge in time domain modeling is to incorporate the frequency dependence in an efficient manner and, as such, several formulations have been proposed. As it will be discussed later in this paper, many of these result in profound modifications of the original model that are sometimes difficult to interpret in the light of their physical meaning. Accounting for frequency related effects is obviously more natural when the governing equations are formulated in the frequency domain rather than in time. However, this implies that incremental time stepping strategies for transient simulation can no longer be applied and harmonic components have to be solved individually, each requiring the solution of a different problem.

In this work we propose a robust parametric solver that allows to determine at once all the harmonics in a given frequency range. While this step is performed "off-line", the actual time response can be efficiently computed "on-line". The rest of this paper is organized as follows, Section 2 presents the literature review. Section 3 presents the method proposed in the current study to deal with the frequency dependent parameters in the frequency domain. In the Section 4 we present the skin effects model for the transmission lines and in Section 5 we present the distributed parameters model of transmission lines that we adopted in this study. Finally, we present the results 
of our proposed approach in the Section 8 .

\section{Transmission Line Modeling}

The frequency dependence of the parameters is the consequence of the assumption made in the derivation of Telegrapher's equations from the Maxwell's equations [6]. It is assumed that the conductor carries a uniform current in a uniform time-harmonic magnetic field. Furthermore, it is assumed that the curvature of the wire is negligible and there is no field variation in the wire direction i.e. $\mathrm{x}$-axis in this case. It is considered that the current flows in x-direction only [7]. These assumptions simplify the 3-D Maxwell's equation to a manageable 1-D equation known as Telegrapher's equation given by:

$$
\begin{aligned}
\frac{\partial v(x, t)}{\partial x} & =-R i(x, t)-L \frac{\partial i(x, t)}{\partial t} \\
\frac{\partial i(x, t)}{\partial x} & =-G v(x, t)-C \frac{\partial v(x, t)}{\partial t} \\
x & \in[0, l] \quad ; \quad t \in[0, \infty]
\end{aligned}
$$

where, $v, i$ are the voltage and current, $R, L, G$ and $C$ are the resistance, inductance, conductance and the capacitance per unit length respectively. Eliminating $i$ and combining the eqs. 1 and 2 yields

$$
L C \frac{\partial^{2} v(x, t)}{\partial t^{2}}+(L G+R C) \frac{\partial v(x, t)}{\partial t}+R G v(x, t)=\frac{\partial^{2} v(x, t)}{\partial x^{2}}
$$

In order to retain the correct physical behavior, the model parameters like resistance and inductance are often considered as function of the frequency in order to compensate for the simplifications introduced by the assumptions 
in the derivation of the 1-D model. The other scenario in which these parameters are kept constant will then require the 3-D partial differential equations to be solved.

Several numerical methods have been proposed for the solution of frequency dependent models [8]. An overview is listed in the Table 1.

There are mainly two families of solution procedures, i.e., a time-domain simulation and a frequency-domain simulation. Both families of methods have their pros and cons and have utility suitable to certain conditions. Timedomain modeling is delicate and some numerical instabilities and accuracy issues have been reported in the literature [5, 9]. But, the advantage it provides is the straightforward compatibility with models for components of the power system.

Most of the studies, reported in Table 1 , avoid convolutions and developed intelligent models to circumvent the difficulties in time-domain modeling by modifying the formulation of the equations in time. For example, in the study by Semlyen and Dabuleanu [10], the authors have used approximations based on the exponential functions. The study by Chu-Sun et al [11] uses equivalent circuit with each section is a combination of equivalent resistances and inductances to include the skin effects. Some recent studies like Marques da Costa et al [3] used fitting methods to include skin effects in lumped parameters model of transmission line.

On the other hand, research studies in favor of frequency-domain modeling point out the ease of formulation. Instead of time convolutions, we can readily multiply the harmonic response of the system with the desired input function. Then obtaining the solution in time-domain is simply just a mat- 
ter of inverse transformation. Wilcox and Condon [12] proposed an inverse transformation method based on ARMA (Auto-Regressive-Moving-Average) fitting method to obtain time-domain model, where ARMA is a statistical model used in several fields to fit model to observed data. While other studies like by Kurokawa et al [1] used modal transformation matrix. In frequencydomain analysis, as described in the study of Meyer and Dommel [13], we have to use linear superposition of all the frequency components. The solution is performed for every individual frequency present in the transients, as the unit impulse response in time, "translates" as the unit heaviside response in frequency-domain.

In the current study, we present a simple yet efficient frequency-domain method based on the Proper Generalized Decomposition for the solution of transmission line including the frequency-dependence of parameters due to skin effects. We propose a method based on PGD's separated representation as a monolithic solver for all the frequencies.

\section{Proposed Approach}

Refering to eq. (3), the second term is referred as the dissipation term and the last term is the dispersion term. For a lossless transmission line we assume $G=0$. By introducing a suitable finite element basis, the function $v(x, t)$ can be approximated as

$$
v(x, t) \approx \sum_{i=1}^{n} v_{i}(t) \phi_{i}(x) .
$$

Then, imposing Galerkin orthogonality of the residual of equation 3 on the basis functions $\phi_{i}(x)$ leads to the following system of ordinary differential 
equations in time

$$
[M]\{\ddot{v}(t)\}+[C]\{\dot{v}(t)\}+[K]\{v(t)\}=\{f(t)\}
$$

where, $[M],[C]$ and $[K]$ are referred to as the mass, damping and stiffness matrices by analogy with structural dynamics. The unknowns of the problems are arranged into the vector $\{v(t)\}=\left\{v_{1}(t), v_{2}(t), \ldots, v_{n}(t)\right\}$. Therefore, $\{\dot{v}(t)\}$ and $\{\ddot{v}(t)\}$ indicate the first and second time derivatives of vector $\{v(t)\}$.

Time integration of Eq. 5 requires the solution of a $n \times n$ algebraic system for every time increment for nonlinear or time dependent matrices. Modal analysis provides an efficient strategy to reduce the numerical complexity of the system by transforming it into a set of $n$ uncoupled equations.

The generalized eigenvalue problem to be solved for the system of eq. (5), with damping $[C]$ removed,

$$
\left(\lambda^{2}[M]+[K]\right)\left\{V_{i}\right\}=\{0\}
$$

where, the vectors $\left\{V_{i}\right\}$ are the eigenvectors and can be grouped in a matrix $[\Lambda]=\left[\left\{V_{1}\right\},\left\{V_{2}\right\}, \cdots,\left\{V_{n}\right\}\right]$. The matrix $[\Lambda]$ can be used to diagonalize the matrices $[M]$ and $[K]$ as:

$$
\begin{aligned}
& {[\Lambda]^{T}[M][\Lambda]=[m]} \\
& {[\Lambda]^{T}[K][\Lambda]=[k]}
\end{aligned}
$$

The system presented in Eq. (5) involves damping, there are generally three ways to proceed further in cases of non-zero damping. The first method is to use the direct time integration which we already explained could require 
solution of $n \times n$ algebraic equations. The second method is to setup and solve a different complex eigensystem in which eigenvalue problem diagonalizes two matrices that contains $[M],[C]$ and $[K]$ as sub-matrices [14]. The final and the easiest method is to continue with the diagonalization presented in eq. (7). For that purpose, we need the damping matrix $[C]$ in a linear combination of the matrices $[M]$ and $[K]$ such that $[C]=a[M]+b[K]$. This is usually the case for the transmission line models because the parameters are constant along the transmission line. Therefore it can also be diagonalized using the same matrix $[\Lambda]$.

$$
[\Lambda]^{T}[C][\Lambda]=a[m]+b[k]
$$

Also, the variable of interest is transformed into the modal basis as:

$$
\{U\}=[\Lambda]^{T}\{V\}
$$

This diagonalization gives us an uncoupled ordinary differential equations that can be solved separately. Writing the equations in indicial notation,

$$
m_{i} \ddot{u}_{i}+\left(a m_{i}+b k_{i}\right) \dot{u}_{i}+k_{i} u_{i}=0
$$

The solution of eq. (9) is straightforward and once we have the solution in terms of modal basis, we can transform the solution back to the physical basis.

$$
\{V\}=[\Lambda]\{U\}
$$

The case we address here is when the parameters resistance $R(\omega)$ and 
inductance $L(\omega)$ becomes frequency dependent because of skin or other effects. The problem where we have frequency-dependent parameters can not be solved needs special attention. Studies such as the one performed by Wilcox and Condon used the modal analysis but modeled the system with frequency-dependent parameters first in frequency-domain and careful manipulation of the system itself is required, otherwise the transformation from frequency-domain model to time-domain model may cause the loss of causality as seen by Crandall [15] in the context of structural dynamics. In his work, Crandall discusses the effect of frequency-dependent parameters on time integration and introduced the concept of non-equations. The better option is to solve the harmonic analysis problem for every frequency $\omega$. Transforming the problem from time-domain to frequency-domain using Fourier transformation of the unknown variable $v_{i}(t)$ and forcing function $f(t)$ that write

$$
\begin{aligned}
& \hat{V}(\omega)=\int_{-\infty}^{\infty} v(t) e^{-\imath \omega t} d t \\
& \hat{F}(\omega)=\int_{-\infty}^{\infty} f(t) e^{-\imath \omega t} d t
\end{aligned}
$$

Therefore, the equation (5) after transformation into frequency-domain is given as:

$$
\left(-\omega^{2}[M(\omega)]+\iota \omega[C(\omega)]+[K(\omega)]\right) \hat{V}(\omega)=\hat{F} \forall \omega
$$

Now, the harmonic analysis problem requires solution of $n$ systems of linear equations where $n$ represent the number of discrete frequencies considered. A similar problem involving fractional damping was discussed in [16]. The use of proper generalized decomposition (PGD) as a paramteric solver 
as presented in earlier studies by Chinesta et al. [17] and [18] is considered here as well. PGD provides a strong tool as a single monolithic solver as compared to solving the harmonic problem using eq. 13 individually for every frequency. PGD has already been implemented for the parametric power flow problem [19] and an error estimation strategy has also been developed [20]. Note that if we solve the linear problem of eq. 13 we have to compute the

matrices for every single frequency as they are no longer constant compared to the case where the constant parameters was considered and depend upon the frequency. Therefore, in this study, we present the Proper Generalized Decomposition (PGD) as a parametric solver, a method capable to solve the harmonic analysis.

\section{Skin Effects Model}

Skin Effect results in frequency dependence of the transmission line parameters. This phenomenon is the result of unequal distribution of current across the cross-section of the transmission line, this is more pronounced in long transmission lines. The parameters that become functions of frequency are resistance and inductance. Inductance of a conductor is related with the flux linkage, the flux linkage in a conductor happens internally and externally. Internal flux linkage is due to the self current of the conductor while the external flux linkage is due to the presence of adjacent conductors. Therefore, total inductance of a transmission line is the sum of inductances due to both internal and external flux linkages. The evaluation of external inductance is based on the assumption that the total current flow is on the surface of the transmission line, i.e., external inductance is maximum when the skin effect is maximum. However, the external inductance is the result of 
the current flow in the neighboring transmission line and therefore remains constant. In the current case, we are dealing with the single transmission line assuming no adjacent transmission lines and in the succeeding discussion wherever inductance is mentioned it is assumed internal inductance unless explicitly mentioned.

Typically transmission lines are bundled aluminum strands twisted together with one kind of transmission line consists of steel strands in the core to increase the strength of the transmission line. However, for the sake of simplicity the discussion is based on a solid cylindrical conductor to effectively demonstrate the model.

Several researchers have presented simple models to include skin effects in the transmission line models [9], [21] and [2]. Since, our focus in the current study is not to model the skin effects but rather use the existing model and present a solution that is able to perform transient simulations with skin effects included. Therefore, we present here some of the simplified models and use them in our study.

For a transmission line with large radius compared to the skin depth [22], $\delta<<4 r$ where skin depth $\delta$ is calculated as (14).

$$
\delta=\left(\frac{2}{\omega \mu \sigma}\right)^{1 / 2}
$$

where, $r$ is the radius of the cross-section of the conductor, $\omega$ is the angular frequency and related to frequency in $\mathrm{Hz}$ as $\omega=2 \pi f, \mu$ is the magnetic permeability and $\sigma$ is the electrical conductivity of the conductor.

The resistance $R(\omega)$ and inductance $L(\omega)$ are given in the study of Lucht [22] based on the expansion of Bessel functions with the assumptions listed 
here. These simplified functions for resistance and inductance for high frequencies is given as:

$$
R(\omega)=\frac{1}{\sigma(2 \pi r) \delta}, \quad L(\omega)=\frac{1}{\sigma(2 \pi r) \delta \omega}
$$

In the technical report by Phil Lucht [22], a low frequency limit is defined, above this frequency resistance and inductance become the functions of frequency, below this limit the resistance and inductance are given by

$$
R=\frac{1}{\sigma \pi r^{2}}, \quad L=\frac{\mu}{8 \pi}
$$

The low frequency resistance is the same as the DC resistance of a conductor in per unit length given by

$$
R=\frac{\rho}{\pi r^{2}}
$$

where $\rho$ is the resistivity of the conducting material.

The article by Monteiro et al [23] presents a simplified formulation for the calculation of skin effects on the transmission line wires. The formulation is based upon the Fourier transformation of the Maxwell's equation [24]. The methodology presented by Monteiro et al is simple in terms of its application without the need to use Bessel functions and also presented good accuracy as it is directly developed from the Maxwell's wave equations.

$$
\begin{aligned}
& R(\omega)=\frac{\sum_{k=1}^{\infty}\left(R_{k} /\left(R_{k}^{2}+\omega^{2} L^{2}\right)\right)}{\left(\sum_{k=1}^{\infty} R_{k} /\left(R_{k}^{2}+\omega^{2} L^{2}\right)\right)^{2}+\omega^{2}\left(\sum_{k=1}^{\infty} L /\left(R_{k}^{2}+\omega^{2} L^{2}\right)\right)^{2}} \\
& L(\omega)=\frac{\sum_{k=1}^{\infty}\left(L /\left(R_{k}^{2}+\omega^{2} L^{2}\right)\right)}{\left(\sum_{k=1}^{\infty} R_{k} /\left(R_{k}^{2}+\omega^{2} L^{2}\right)\right)^{2}+\omega^{2}\left(\sum_{k=1}^{\infty} L /\left(R_{k}^{2}+\omega^{2} L^{2}\right)\right)^{2}}
\end{aligned}
$$




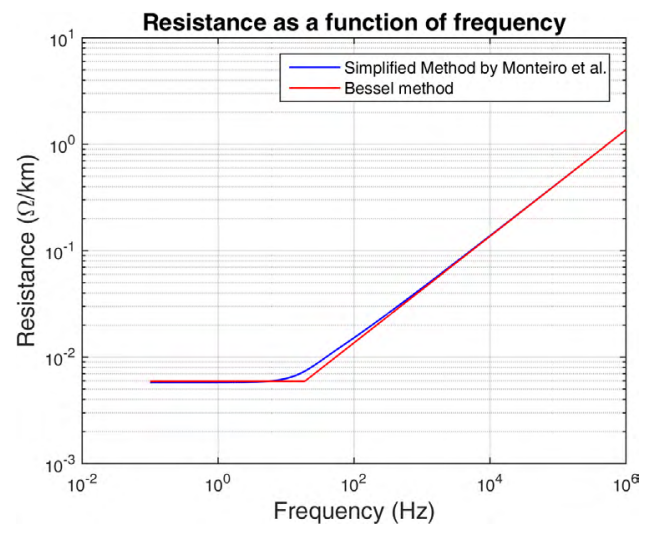

(a) Step Response

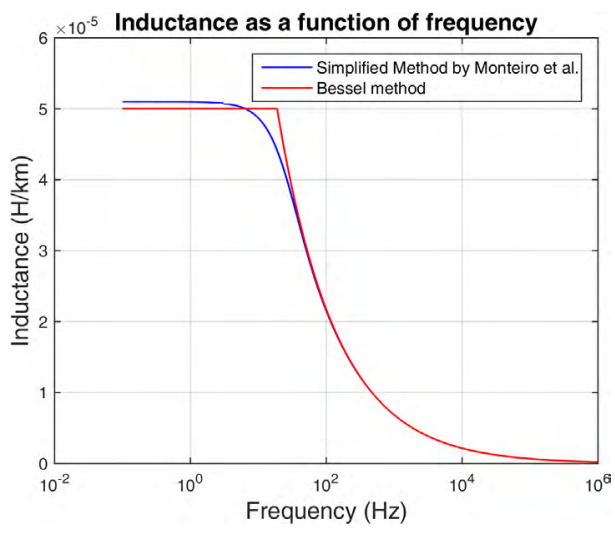

(b) Sine Response

Figure 1: Frequency dependent resistance and inductance of a conductor

where

$$
R_{k}=\frac{\xi_{k}^{2}}{4 \pi \sigma r^{2}} ; \quad L=\frac{\mu}{4 \pi} \quad \text { and } \quad \xi_{k}=\frac{(2 k-1) \pi}{2}+\frac{\pi}{4}
$$

The units of $R_{k}$ is $\Omega . m$ and $L$ is $H / m$. The units of electrical conductivity $\sigma$ is $S / m$ and of the magnetic permeability $\mu$ is $H / m$. The term $\xi_{k}$ is the $k^{\text {th }}$ Bessel function root and is directly proportional to $k$. As mentioned previously, the transmission line in this study is considered to be a solid cylindrical copper wire, its radius and the electrical properties of the copper are listed in Table 2 .

A comparison of different models was performed and the results were compared with the results from the study of Monteiro et al [23]. The results for the resistance and inductance with respect to the frequency is presented in the Figures $1 \mathrm{a}$ and $1 \mathrm{~b}$. 


\section{Distributed Parameters Model}

\subsection{Time Domain Simulation}

We start with the time domain distributed parameters (DP) model. The electrical transmission lines provide the path for the voltage and current waves to propagate, the dynamics of the propagation is described by a distributed parameters model. This model depends upon the considerations taking into account like the losses and the frequency dependence of the parameters.

The distributed parameters model for a lossy transmission line with constant parameters can be written in the time domain as:

$$
\begin{aligned}
& \frac{\partial v(x, t)}{\partial x}=-L \frac{\partial i(x, t)}{\partial t}-R i(x, t), \\
& \frac{\partial i(x, t)}{\partial x}=-C \frac{\partial v(x, t)}{\partial t}
\end{aligned}
$$

for $0 \leq x \leq l$, where $v(x, t)$ and $i(x, t)$ are the voltage and current in the line respectively, and the resistance, the inductance and the capacitance are represented by $R, L, C$ respectively and these constants are positive and independent of frequency. The parameters $R, L, C$ are given in per unit length and in distributed parameters model are used without multiplication with the length of the line. However, these parameters must be multiplied by the length of the line in case one is using any lumped circuit model for transmission lines e.g. pi-equivalent model. The effect of transverse conductance $G$ of the line is neglected in this model. The interconnection between the transmission line, the load and the generator is performed using the Kirschhoff's laws and the boundary values. 


$$
\begin{aligned}
& \left.v(x, t)\right|_{x=0}=V_{0}(t) \\
& \left.i(x, t)\right|_{x=l}=0
\end{aligned}
$$

The term $V_{0}$ is the voltage source assumed to represent a generator, the boundary condition given above assumes that the voltage source is ideal, that is, the impedance is zero $\left(Z_{0}=0\right)$ and the impedance at the receiving end $(x=l)$ is infinite $\left(Z_{l}=\infty\right)$.

The equation is converted to a single variable second order differential equation in time and space.

$$
\frac{\partial^{2} v}{\partial x^{2}}-L C \frac{\partial^{2} v}{\partial t^{2}}-R C \frac{\partial v}{\partial t}=0
$$

The equation $(23)$ is then discretized in time and space, using finite difference scheme for the time integration and finite elements for the space.

For a copper conductor wire, we can calculate the resistance and inductance from the equations (16), these values are listed in the table 3 . Solving a space time problem for these values, with a boundary condition representing a step as an input voltage $V_{0}$.

$$
V_{0}=\left\{\begin{array}{l}
0 \text { for } t \leqslant 0 \\
1 \text { for } t>0
\end{array}\right.
$$

Also, a sine function with a frequency of $f=100 \mathrm{~Hz}$ was used as an input voltage for the same system

$$
V_{0}=\left\{\begin{array}{c}
0 \text { for } t \leqslant 0 \\
\sin (2 \pi 100 t) \text { for } t>0
\end{array}\right.
$$




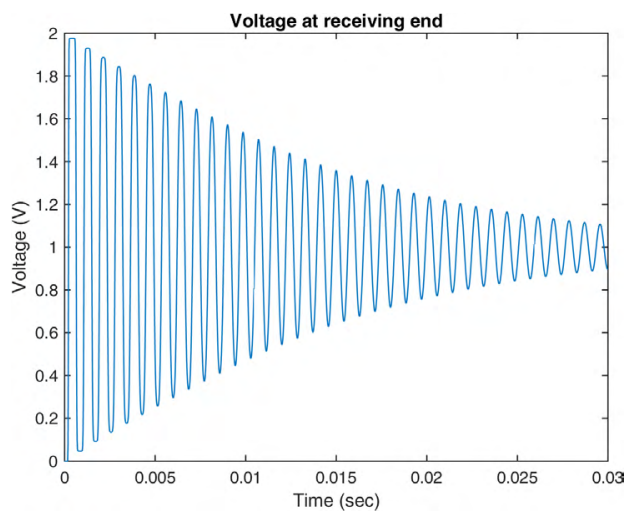

(a) Step Response

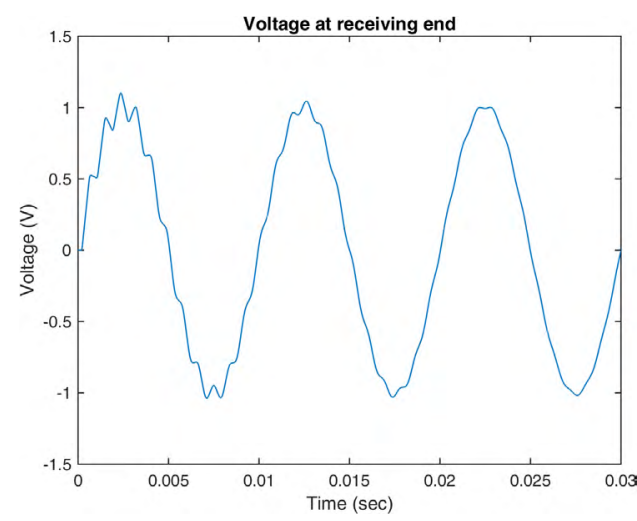

(b) Sine Response

Figure 2: Time simulation of constant parameters model in space-time domain

The results of time simulation for the receiving end voltage $x=l$ is presented graphically in the Figures $2 \mathrm{a}$ and $2 \mathrm{~b}$.

\section{Frequency-Domain Model}

Skin effects phenomena results in frequency dependence of DP model, direct time integration of which is difficult to perform. But, the problem in the frequency domain becomes a linear second order differential equation, where the parameters resistance and inductance are now a function of frequency. Fourier transformation of the time domain distributed parameters model gives the equation in the frequency domain as:

$$
\begin{aligned}
& \frac{\partial \hat{V}(x, \omega)}{\partial x}=-\hat{I}(x, \omega) \cdot(R+\iota L \omega), \\
& \frac{\partial \hat{I}(x, \omega)}{\partial x}=-\hat{V}(x, \omega) \cdot(\iota C \omega)
\end{aligned}
$$

Combining the two equations given in (26), we get a second order ordinary differential equations for voltage. 


$$
\frac{\partial^{2} \hat{V}(x, \omega)}{\partial x^{2}}+\left(L C \omega^{2}-\iota R C \omega\right) \hat{V}(x, \omega)=0
$$

Corresponding boundary conditions in the frequency domain is given as

$$
\begin{aligned}
& \left.\hat{V}(x, \omega)\right|_{x=0}=V_{0} \\
& \left.\frac{\partial \hat{V}(x, \omega)}{\partial x}\right|_{x=l}=0
\end{aligned}
$$

\section{Proper Generalized Decomposition for Constant Parameters Model}

The problem presented in Sec. 5 can be solved using PGD that assures the separated representation,

$$
\hat{V}(x, \omega)=\sum_{i=1}^{m} X_{i}(x) . O_{i}(\omega)
$$

For the details about PGD formulation and its advantage, one can refer to several publications for example, [18, 17]. In general, PGD methods comprises of an offline and an online part. In the offline stage of the solution, the problem is formulated in the separated representation and the solution database is created. The online part then is just to compute the solution for the particular problem by multiplying the separated coordinates for each enrichment mode.

Once, a solution using PGD with the boundary conditions given in (28) is obtained, it is straightforward to get the desired time response using the discrete inverse Fourier function available in MATLAB. Using the separated representation of (29), the DP model can be expressed in separated repre- 
sentation. In order to develop a PGD formulation, first we must have a weak formulation of the problem.

Multiply equation (27) with a test function $\delta V^{*}(x)$ where, $\delta V$ represents the test function and $\delta V^{*}(x)$ represents the complex conjugate.

$$
\int_{\Omega_{x} \times \Omega_{\omega}} \delta V^{*} \frac{\partial^{2} \hat{V}}{\partial x^{2}} d x d \omega-\int_{\Omega_{x} \times \Omega_{\omega}} \delta V^{*} f(\omega) \hat{V} d x d \omega=0
$$

where, $f(\omega)=\left(L C \omega^{2}-\iota R C \omega\right)$.

Here, we briefly describe the PGD algorithm, for details the interested readers can refer to the literature [17, 18]. PGD follows the greedy algorithm approach, i.e., one mode is evaluated at a time. The mode is assumed to be converged after sufficient iterations result in no further improvement. This procedure of adding modes is termed as the enrichment process. Assuming that $n-1$ initial modes are already converged, we define the $n^{\text {th }}$ mode to be evaluated as:

$$
\hat{V}(x, \omega)=\sum_{i=1}^{n-1} X_{i}(x) \cdot O_{i}(\omega)+X_{n}^{p}(x) O_{n}^{p-1}(\omega)
$$

We assume the test function $\delta V^{*}(x, \omega)$ in the separated form as

$$
\delta V^{*}(x, \omega)=O^{*}(\omega) \delta X^{*}(x)+X^{*}(x) \delta O^{*}(\omega)
$$

In eq. (31), the superscript $p$ indicates the number of fixed point iterations. For individual modes evaluation, we apply the fixed point loop which is to find solution for each coordinate space alternatively.

Since, the problem is now separated in the space $X(x)$ and the frequency $O(\omega)$, we have to solve two problems using FEM once for the space domain 
and then for the frequency domain. The weak formulation for the space only problem is:

$$
\begin{aligned}
& \int_{\Omega_{x} \times \Omega_{\omega}} \frac{d \delta X_{n}^{*}}{d x} \frac{d X_{n}^{p}}{d x} O_{n}^{p-1 *} O_{n}^{p-1} d x d \omega+\int_{\Omega_{x} \times \Omega_{\omega}} f(\omega) \delta X_{n}^{*} X_{n}^{p} O_{n}^{p-1 *} O_{n}^{p-1} d x d \omega= \\
& -\int_{\Omega_{x} \times \Omega_{\omega}} \sum_{i=1}^{n-1} \frac{d \delta X_{n}^{*}}{d x} \frac{d X_{i}}{d x} O_{n}^{p-1 *} O_{i} d x d \omega-\int_{\Omega_{x} \times \Omega_{\omega}} f(\omega) \sum_{i=1}^{n-1} \delta X_{n}^{*} X_{i} O_{n}^{p-1 *} O_{i} d x d \omega \\
& +\int_{\Omega_{\omega}} \delta X_{n}^{*} O_{n}^{p-1 *} \hat{I}(\iota L \omega+R) d \omega
\end{aligned}
$$

and for the problem to be solved in the frequency domain, the weak formulation will be

$$
\begin{aligned}
& \int_{\Omega_{x} \times \Omega_{\omega}} \frac{d X_{n}^{p *}}{d x} \frac{d X_{n}^{p}}{d x} \delta O_{n}^{*} O_{n}^{p} d x d \omega+\int_{\Omega_{x} \times \Omega_{\omega}} f(\omega) X_{n}^{p *} X_{n}^{p} \delta O_{n}^{*} O_{n}^{p} d x d \omega= \\
& -\int_{\Omega_{x} \times \Omega_{\omega}} \sum_{i=1}^{n-1} \frac{d X_{n}^{p *}}{d x} \frac{d X_{i}}{d x} \delta O_{n}^{*} O_{i} d x d \omega-\int_{\Omega_{x} \times \Omega_{\omega}} f(\omega) \sum_{i=1}^{n-1} X_{n}^{p *} X_{i} \delta O_{n}^{*} O_{i} d x d \omega \\
& +\left.\int_{\Omega_{\omega}} X_{n}^{p *}\right|_{x=0} \delta O_{n}^{*} \hat{I}(\iota L \omega+R) d \omega
\end{aligned}
$$

The tolerances selected for the stopping of the fixed point algorithm $\varepsilon_{p}$ and the tolerance for termination of enrichment process $\varepsilon_{n}$, along with the number of modes $n$ in both the constant parameters and frequency-dependent parameters are listed in Table 4 .

The boundary condition in eq $(28)$ is an equivalent of having an impulse function at the source which gives the impulse response or the transfer function $\hat{H}$ of the transmission line. The voltage impulse response at the receiving end is given as: 


$$
\hat{H}(x=l, \omega)=\sum_{i=1}^{n} X_{i}(x=l) O_{i}(\omega)
$$

Once, this is done, the time response to any arbitrary condition at the source node is then straightforward multiplication of the voltage function in frequency domain with the particular solution of the PGD at the receiving node. Then the frequency response to an arbitrary function $\hat{V}_{0}$ is given as

$$
\hat{V}(x=l, \omega)=\hat{H}(x=l, \omega) \cdot \hat{V}_{0}
$$

The input voltage $\hat{V}_{0}$ is usually a step function or a sinusoidal function in time. In practice, these functions are transformed into frequency-domain using the fast Fourier transform algorithm to compute the discrete Fourier transform. Similarly, the response $\hat{V}$ is inverse transformed to time domain using the same fast Fourier algorithm using discrete samples in the frequency interval.

\subsection{Verification of $P G D$ results}

In this section, we first present the time response using inverse fourier transform of the PGD solution particularized at the receiving end.

The PGD solution in terms of voltage on a space-frequency domain is presented in Figures $3 \mathrm{a}$ to $3 \mathrm{c}$.

The results from Figures $5 \mathrm{a}$ and $5 \mathrm{~b}$ shows good correlation between the solution from time integration and the PGD solution. Therefore, it is established that the PGD solution is an accurate representation of the DP model of transmission lines. The difference between the two curves of in the Figure $5 \mathrm{a}$ is due to the fact that the time simulation is carried out using 1st 


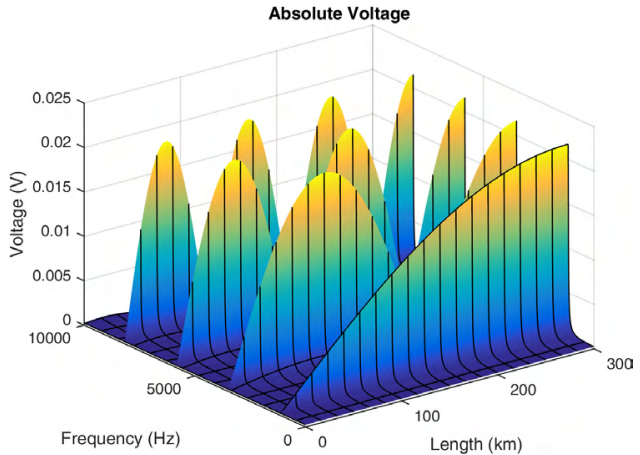

(a) Absolute Value

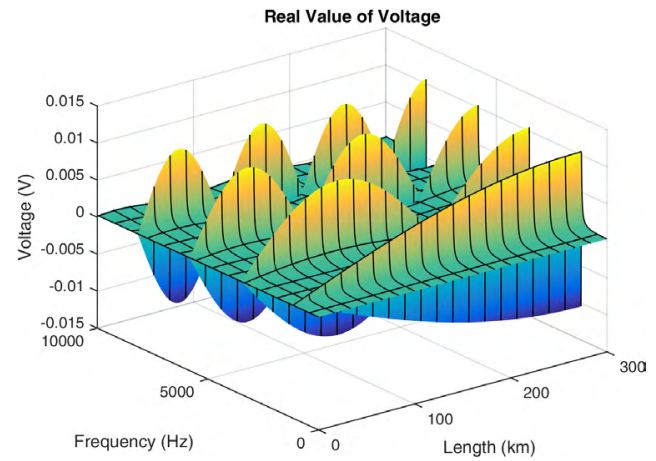

(b) Real Value

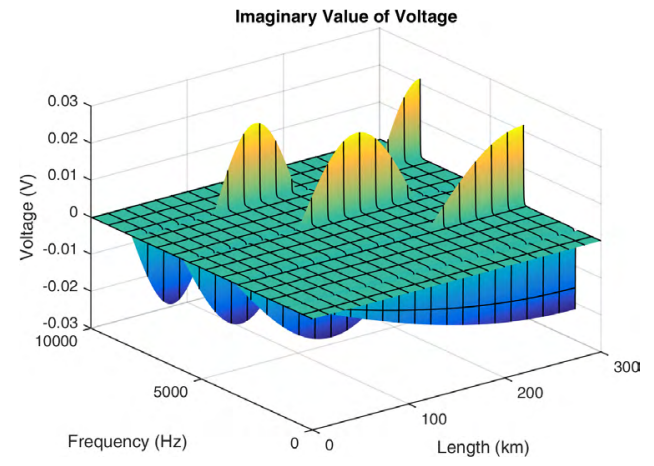

(c) Imaginary Value

Figure 3: Voltage in space-frequency domain with constant parameters

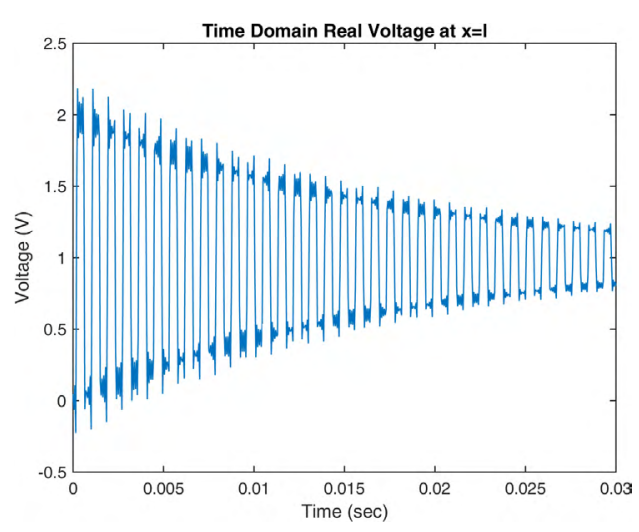

(a) Step Response

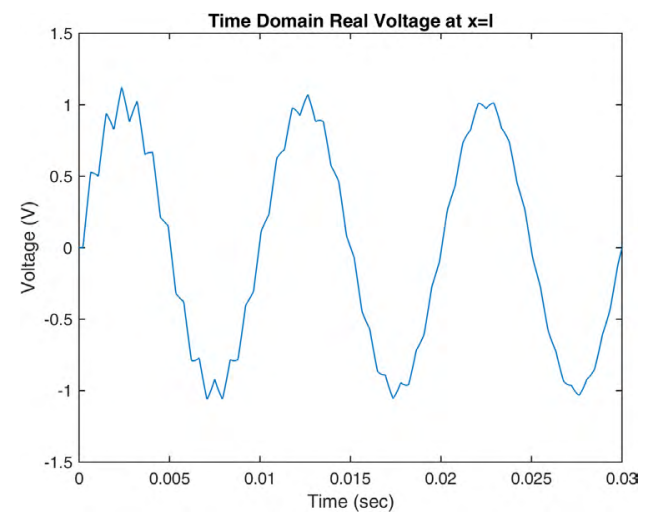

(b) Sine Response

Figure 4: Time response by inverse Fourier of the PGD solution 


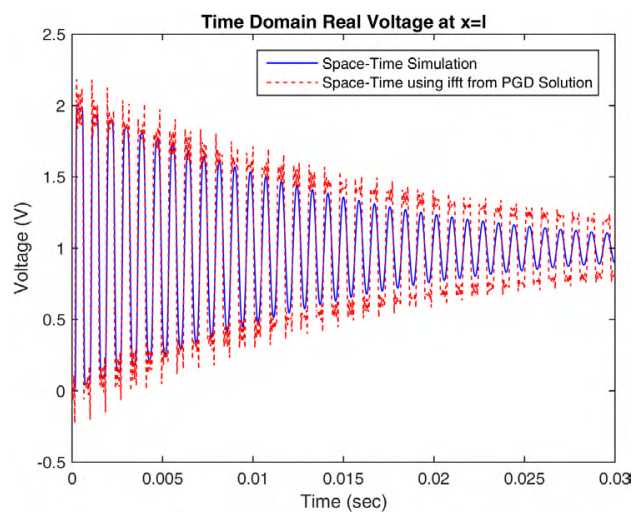

(a) Step Response

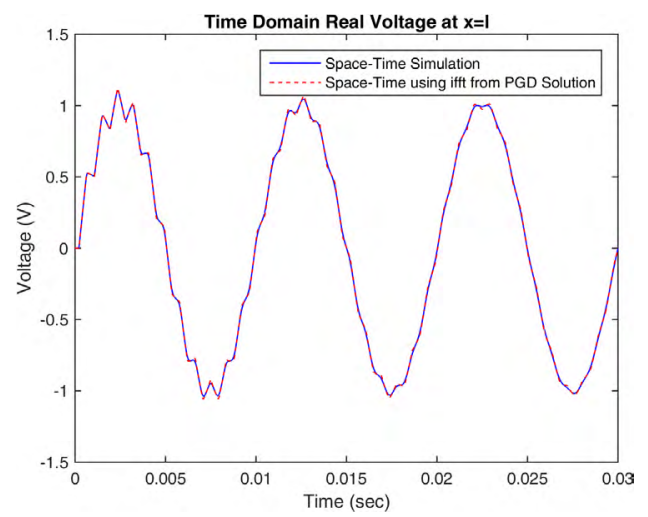

(b) Sine Response

Figure 5: Comparison of time response between PGD solution and time integration

order backward difference scheme. The blue curve of time simulation tends to approach the red curve of PGD solution as finer time discretization is used but even then spikes observed in the PGD solution are not captured by the time simulation. In comparison, PGD based approach uses frequency domain which by default is a higher order approximation and hence able to capture a wider range of dynamics.

\section{PGD for frequency dependent parameters}

The benefit of solving DP model in the frequency domain in the separated representation of PGD is the easy, fast and accurate solution with the option of a parametric solution. PGD methods provide the best option for parametric problems both in terms of computational efficiency and ease of solving the problem. In the case of skin effects, the equation (27) now has parameters resistance $R$ and inductance $L$ as a function of frequency $\omega$. As explained earlier time integration of such problems is almost impossible to achieve and hence PGD provides real accurate option to solve such problems. 
The problem is similar to the case of fractional derivatives which is similar to the fractional RLC circuit [25].

Eq 277) with $\mathrm{R}$ and $\mathrm{L}$ as functions of frequency reads as:

$$
\frac{\partial^{2} \hat{V}(x, \omega)}{\partial x^{2}}+\left(L(\omega) \cdot C \cdot \omega^{2}-\iota R(\omega) \cdot C \cdot \omega\right) \hat{V}(x, \omega)=0
$$

The increase in the resistance with the frequency and a simultaneous decrease in the inductance results in a system more damped than the system with constant resistance and inductance. This effect can be noticed in the Figures 6a to 6c showing the voltage in the space-frequency domain, there is only one peak visible with a much less amplitude.

The effect of increased damping due to the increase in resistance can be compared in the receiving end voltage for the frequency dependent and constant paramters solution given in the Figures 7. In Figure 8, the two responses from the constant parameters and frequency dependent parameters are plotted on top of each other for a direct comparison. The increased damping removes almost all the oscillations observed in the constant parameters solution of Figures $4 \mathrm{a}$ and $4 \mathrm{~b}$. The difference between the constant parameters model and frequency dependent parameters solution are very different. Therefore, just to elaborate the effect of different values of the parameters we used the constant parameters model with parameters values evaluated at $60 \mathrm{~Hz}$ and $120 \mathrm{~Hz}$ and plotted in the Figure 9.

\subsection{Parametric Solution}

The advantage of PGD based harmonic analysis is that any parameter can be introduced as a model extra-coordinate into the separated representation. 


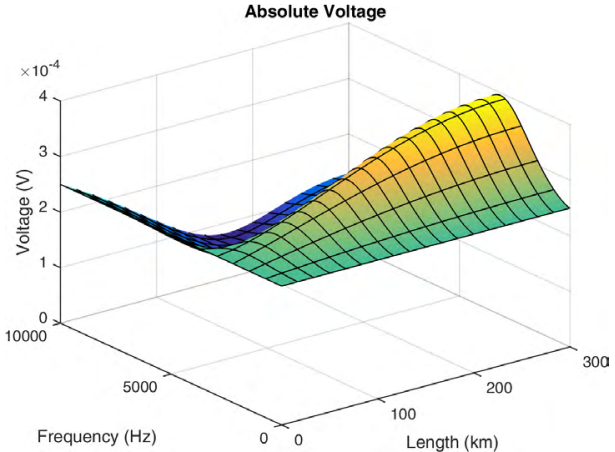

(a) Absolute Value

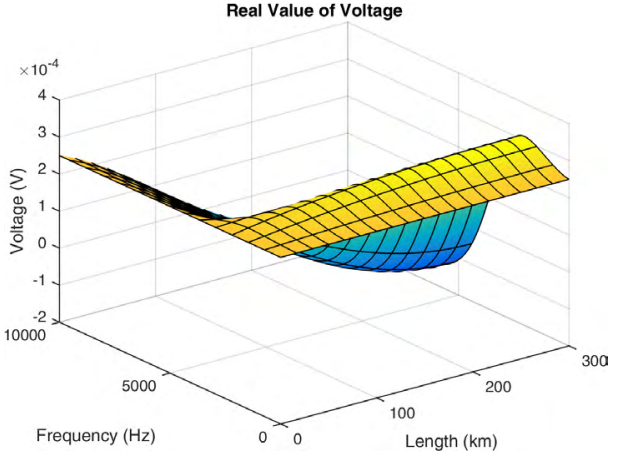

(b) Real Value

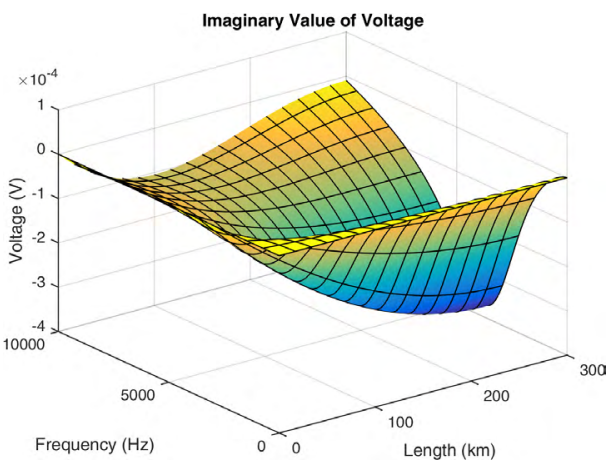

(c) Imaginary Value

Figure 6: Voltage in space-frequency domain with skin effects included

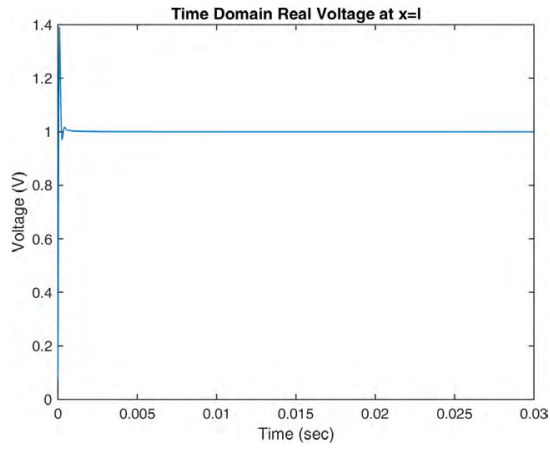

(a) Step Response

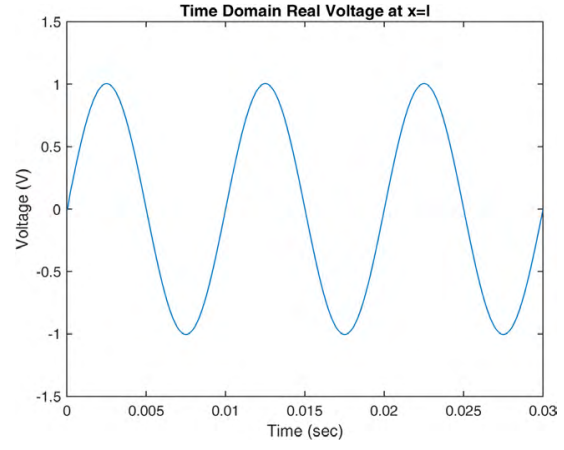

(b) Sine Response

Figure 7: Time response from PGD solutions for frequency dependent parameters 


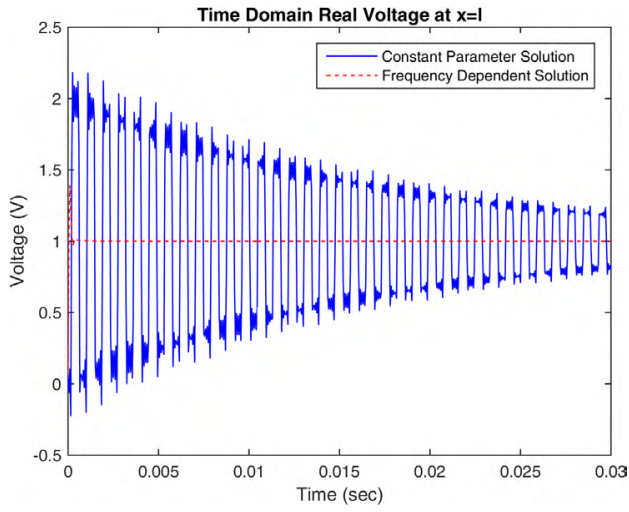

(a) Step Response

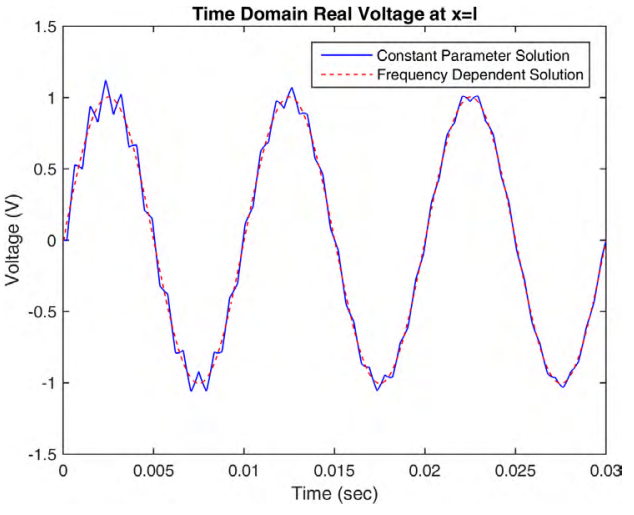

(b) Sine Response

Figure 8: Comparison of response between constant and frequency dependent parameters

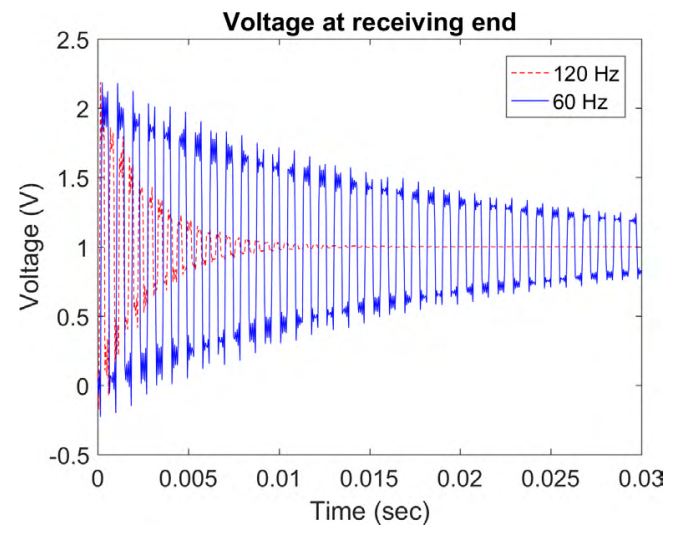

(a) Step Response

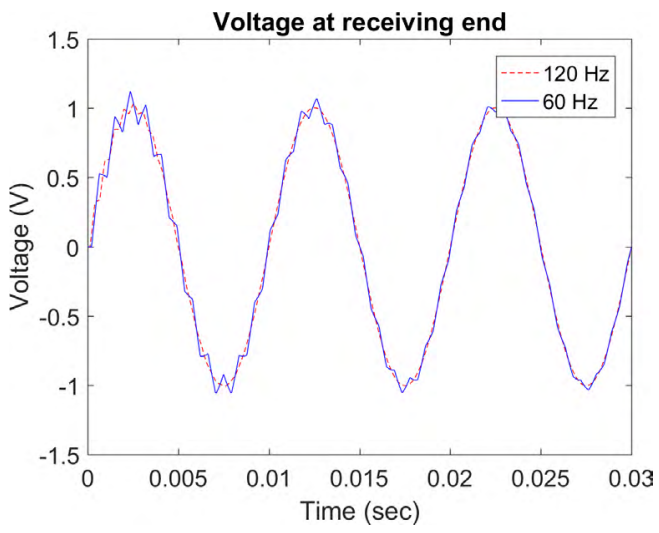

(b) Sine Response

Figure 9: Comparison of response for constant parameters with parameter values at 60 $\mathrm{Hz}$ and $120 \mathrm{~Hz}$ 
As an initial step, we introduce length of the transmission line as a new dimension for the parametric problem. The solution now is given as:

$$
\hat{V}(x, \omega, l)=\sum_{i=1}^{n} X_{i}(x) O_{i}(\omega) D_{i}(l)
$$

where $D_{i}$ represents the modes of the dimension introduced to represent length.

The mathematical formulation of the problem remains similar and there is no significant additional computational cost to the problem. For the case, we have reported in this study, the new dimension of transmission line length is considered for a range $(200,300) \mathrm{km}$. The discretization for the space and frequency have been kept the same as in previous case. The discretization and the other information valuable to gauge the PGD solution is listed in Table 5 .

The advantage of parametric solution is presented in Figure 10 where the variation of voltages along the transmission line with different lengths for a frequency of $60 \mathrm{~Hz}$ is plotted. Another advantage of such a solution is in the sensitivity or uncertainty analysis, in such an analysis a fixed point is selected and a variation of usually $5 \%$ in the independent variable and its effect on the variable of interest is studied. With a PGD solution, we already have a complete solution as a curve and the derivative can be easily evaluated for the sensitivity analysis.

\section{Conclusions}

The work presented in this study, shows the effectiveness of frequencydomain methods for problems involving frequency-dependent parameters. 


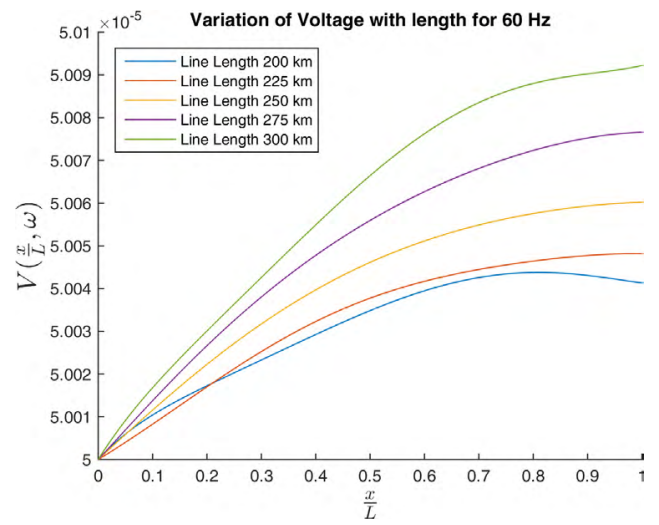

Figure 10: Variation of voltage along the transmission line for different lengths at $60 \mathrm{~Hz}$

The main contribution of the study presented in this paper is the development of a reduced order model able to include phenomenon like skin effects that cause the parameters to become functions of frequency. The method is equally applicable for the simulation of transmission line models including the ground return and proximity effects which has a similar effect on the parameters, i.e., the frequency dependence. In particular, harmonic analysis using PGD is fast, accurate and simple method for this kind of parametric problem. In this paper, we have demonstrated the method of PGD, constructing the database of the parametric solution offline and particularize the solution online for a specific set of parameters online in real time. PGD is an a-priori model order reduction method and is particularly effective to obtain solutions online from the pre-computed database of solutions. With the ability of including parameters such as the length of the transmission line or material properties of the conducting material as one of the coordinates of the separated representation, it truly provides the capability of having real time solutions using not so power platforms. In contrast to the 
method presented in the current study, there are solution methods available that include the frequency-dependence of parameters and simulate the model in time-domain, but these methods are complicated to implement and since they use time integration it is computationally costly and time consuming. PGD is already established as a fast and accurate method for reduced order modeling. Another potential application of this method could be fractional RLC circuits.

\section{References}

[1] Sérgio Kurokawa, J. Pissolato Filho, M.C. Tavares, C.M. Portela, and A.J. Prado. Behavior of Overhead Transmission Line Parameters on the Presence of Ground Wires. IEEE Transactions on Power Delivery, 20(2):1669-1676, apr 2005.

[2] M. Admane, M. Sorine, and Q. Zhang. Simulation of electric transmission lines with skin effect based on a reduced order differential model. In Reliability in Electromagnetic Systems, 2007 IET Colloquium on, pages $1-5$, May 2007.

[3] Eduardo Coelho Marques da Costa, Sérgio Kurokawa, Afonso José do Prado, and José Pissolato. Proposal of an alternative transmission line model for symmetrical and asymmetrical configurations. International Journal of Electrical Power \& Energy Systems, 33(8):1375-1383, oct 2011 .

[4] Dierk Bormann and Hanif Tavakoli. Reluctance Network Treatment 
of Skin and Proximity Effects in Multi-Conductor Transmission Lines. IEEE Transactions on Magnetics, 48(2):735-738, feb 2012.

[5] J. R. Marti. Accurate Modeling of Frequency-Dependent Transmission Lines in Electromagnetic Transient Simulations. IEEE Transactions on Power Apparatus and Systems, PAS-101(1):147-157, 1982.

[6] C.A. Nucci, F. Rachidi, and M. Rubinstein. Derivation of telegrapher's equations and field-to-transmission line interaction. In Electromagnetic Field Interaction with Transmission Lines, volume 29, pages 3-22. WIT Press, feb 2008.

[7] Hajime Igarashi. Semi-Analytical Approach for Finite Element Analysis of Multi-turn Coil Considering Skin and Proximity Effects. IEEE Transactions on Magnetics, 02(c):1-1, 2016.

[8] V Maló Machado, M Eduarda Pedro, J.A. Brandão Faria, and D. Van Dommelen. Magnetic field analysis of three-conductor bundles in flat and triangular configurations with the inclusion of proximity and skin effects. Electric Power Systems Research, 81(11):2005-2014, nov 2011.

[9] Kyung Suk Oh. Accurate transient simulation of transmission lines with the skin effect. IEEE Transactions on Computer-Aided Design of Integrated Circuits and Systems, 19(3):389-396, mar 2000.

[10] A. Semlyen and A. Dabuleanu. Fast and accurate switching transient calculations on transmission lines with ground return using recursive convolutions. IEEE Transactions on Power Apparatus and Systems, 94(2):561-571, Mar 1975. 
[11] Chu-Sun Yen, Zvonko Fazarinc, and R.L. Wheeler. Time-domain skineffect model for transient analysis of lossy transmission lines. Proceedings of the IEEE, 70(7):750-757, 1982.

[12] D.J. Wilcox and M. Condon. A new transmission-line model for timedomain implementation. COMPEL - The international journal for computation and mathematics in electrical and electronic engineering, 16(4):261-274, dec 1997.

[13] W. S. Meyer and H. W. Dommel. Numerical modelling of frequencydependent transmission-line parameters in an electromagnetic transients program. IEEE Transactions on Power Apparatus and Systems, PAS93(5):1401-1409, Sept 1974.

[14] R R Craig Jr. and A J Kurdila. Fundamentals of Structural Dynamics. John Wiley \& Sons, Ltd, New Jersey, 2006.

[15] S.H. Crandall. The role of damping in vibration theory. Journal of Sound and Vibration, 11(1):3 - IN1, 1970.

[16] C. Germoso, A. Fraile, E. Alarcon, J.V. Aguado, and F. Chinesta. From standard to fractional structural visco-elastodynamics: Application to seismic site response. Physics and Chemistry of the Earth, Parts $A / B / C$, pages -, 2016.

[17] F. Chinesta, A. Ammar, A. Leygue, and R. Keunings. An overview of the proper generalized decomposition with applications in computational rheology. Journal of Non-Newtonian Fluid Mechanics, 166(11):578-592, 2011. 
[18] F. Chinesta, A. Leygue, F. Bordeu, J. V. Aguado, E. Cueto, D. Gonzalez, I. Alfaro, A. Ammar, and A. Huerta. PGD-Based Computational Vademecum for Efficient Design, Optimization and Control. Archives of Computational Methods in Engineering, 20(1):31-59, 2013.

[19] R. García-Blanco, P. Díez, D. Borzacchiello, and F. Chinesta. A reduced order modeling approach for optimal allocation of distributed generation in power distribution systems. In 2016 IEEE International Energy Conference (ENERGYCON), pages 1-6, April 2016.

[20] R. García-Blanco, D. Borzacchiello, F. Chinesta, and P. Díez. Monitoring a pgd solver for parametric power flow problems with goal-oriented error assessment. International Journal for Numerical Methods in Engineering, pages $\mathrm{n} / \mathrm{a}-\mathrm{n} / \mathrm{a}, 2016$. nme.5470.

[21] O.M.O Gatous and J Pissolato. Frequency-dependent skin-effect formulation for resistance and internal inductance of a solid cylindrical conductor. IEE Proceedings - Microwaves, Antennas and Propagation, 151(3):212, 2004.

[22] Phil Lucht. Transmission Lines and Maxwell's Equations. Technical report, 2014.

[23] José Humberto A Monteiro, Eduardo Coelho M Costa, André Jinno G Pinto, Sérgio Kurokawa, Omar Mohamed O Gatous, and José Pissolato. Simplified skin-effect formulation for power transmission lines. IET Science, Measurement \& Technology, 8(2):47-53, mar 2014. 
[24] Robert A. Chipman. Theory and Problems of Transmission Lines. McGraw-Hill, 1968.

[25] Gómez-Aguilar José Francisco, Rosales-García Juan, Guía-Calderón Manuel, and Razo-Hernández José Roberto. Fractional RC and LC electrical circuits. Ingeniería, Investigación y Tecnología, 15(2):311 $319,2014$.

[26] M. Davila, J. L. Naredo, P. Moreno, and A. Ramirez. Practical implementation of a transmission line model for transient analysis considering corona and skin effects. In Power Tech Conference Proceedings, 2003 IEEE Bologna, volume 2, pages 6 pp. Vol.2-, June 2003.

[27] B. Gustavsen, J. Sletbak, and T. Henriksen. Calculation of electromagnetic transients in transmission cables and lines taking frequency dependent effects accurately into account. IEEE Transactions on Power Delivery, 10(2):1076-1084, apr 1995.

[28] S Kim and D.P. Neikirk. Compact equivalent circuit model for the skin effect. In 1996 IEEE MTT-S International Microwave Symposium Digest, volume 3, pages 1815-1818. IEEE, 1996. 


\begin{tabular}{|c|c|c|}
\hline Authors & Phenomena & Methods \\
\hline \multicolumn{3}{|c|}{ Time Domain Modeling } \\
\hline Semlyn and & & Approximation of time variable \\
\hline $\begin{array}{l}\text { Dabuleanu } \\
{[10]}\end{array}$ & Ground return effect & $\begin{array}{l}\text { characteristic admittance using expo- } \\
\text { nential functions }\end{array}$ \\
\hline J. R. Marti [5] & Ground return effect & $\begin{array}{l}\text { Weighting Functions, } \\
\text { Characteristic Impedance }\end{array}$ \\
\hline Chu-Sun et al 11 & Skin effect & $\begin{array}{l}\text { Equivalent Circuit using parallel } \\
\text { equivalent resistors and inductors }\end{array}$ \\
\hline Suk Oh 9$]$ & Skin effect & Difference Approximation Method \\
\hline $\begin{array}{l}\text { Marques da } \\
\text { Costa et al } 3]\end{array}$ & $\begin{array}{l}\text { Ground return, } \\
\text { skin and corona } \\
\text { effects }\end{array}$ & $\begin{array}{l}\text { Fitting Procedure for state space } \\
\text { lumped parameters models }\end{array}$ \\
\hline Dávila et al [26] & $\begin{array}{l}\text { Corona and Skin } \\
\text { Effects }\end{array}$ & $\begin{array}{l}\text { Differential Integral Equation } \\
\text { (Radulet Line Equations) }\end{array}$ \\
\hline \multicolumn{3}{|c|}{ Frequency Domain Modeling } \\
\hline $\begin{array}{l}\text { Meyer and } \\
\text { Dommel [13] }\end{array}$ & Ground return effect & $\begin{array}{l}\text { Linear Superposition } \\
\text { of all frequency components } \\
\text { Modal Analysis, }\end{array}$ \\
\hline $\begin{array}{l}\text { Wilcox and } \\
\text { Condon }[12]\end{array}$ & Skin Effects & $\begin{array}{l}\text { Conversion to time-domain using Auto- } \\
\text { Regressive-Moving-Average (ARMA) } \\
\text { as fitting method }\end{array}$ \\
\hline Gustavsen et al [27] & $\begin{array}{l}\text { Frequency de- } \\
\text { pendent effects } \\
\text { of underground } \\
\text { cables }\end{array}$ & $\begin{array}{l}\text { Travelling wave model, } \\
\text { Recursive Convolutions }\end{array}$ \\
\hline Kurokawa et al [1] & Ground return effect & $\begin{array}{l}\text { Clarke's matrix as the } \\
\text { modal transformation matrix }\end{array}$ \\
\hline Machado et al. [8] & $\begin{array}{l}\text { Skin and Prox- } \\
\text { imity Effects }\end{array}$ & $\begin{array}{l}\text { Magnetic Field Analysis using } \\
\text { Bessel function development }\end{array}$ \\
\hline $\begin{array}{l}\text { Bormann and } \\
\text { Tavakoli [4] }\end{array}$ & $\begin{array}{l}\text { Skin and Prox- } \\
\text { imity Effects }\end{array}$ & Reluctance networks \\
\hline $\begin{array}{l}\text { Gatous and } \\
\text { Pissolato 21] }\end{array}$ & Skin Effects & $\begin{array}{l}\text { Simplified expressions of } R \text { and } L \\
\text { based on Bessel Functions }\end{array}$ \\
\hline $\begin{array}{l}\text { Kim and } \\
\text { Neikirk [28] }\end{array}$ & Skin Effects & $\begin{array}{l}\text { Compact Circuit Model } \\
\text { in form of Ladder Circuit }\end{array}$ \\
\hline $\begin{array}{l}\text { Admane et al } \\
{[2]}\end{array}$ & Skin Effects & $\begin{array}{l}\text { Reduced order differential model } \\
\text { based on Kim's Ladder Circuit }\end{array}$ \\
\hline
\end{tabular}

Table 1: Literature review 


\begin{tabular}{lcc}
\hline Parameter & Value & Units \\
\hline Radius (r) & 30 & $\mathrm{~mm}$ \\
Length $(\mathrm{l})$ & 300 & $\mathrm{~km}$ \\
Electrical Conductivity $(\sigma)$ & $5.96 \times 10^{7}$ & $\mathrm{~S} / \mathrm{m}$ \\
Magnetic Permeability $(\mu)$ & $1.26 \times 10^{-6}$ & $\mathrm{H} / \mathrm{m}$ \\
\hline
\end{tabular}

Table 2: Copper conductor wire properties 


\begin{tabular}{lcc}
\hline Parameter & Value & Units \\
\hline Resistance $(\mathrm{R})$ & $5.7603 \times 10^{-3}$ & $\mathrm{ohm} / \mathrm{km}$ \\
Inductance $(\mathrm{L})$ & $5.0980 \times 10^{-5}$ & $\mathrm{H} / \mathrm{km}$ \\
Capacitance $(\mathrm{C})$ & $1.00 \times 10^{-8}$ & $\mathrm{~F} / \mathrm{km}$ \\
Length $(\mathrm{l})$ & 300 & $\mathrm{~km}$ \\
Space Discretization $(\Delta x)$ & $1.0 \times 10^{-1}$ & $\mathrm{~km}$ \\
Total Time $(\mathrm{T})$ & $3.0 \times 10^{-2}$ & $\mathrm{sec}$ \\
Time Step $(\Delta t)$ & $1.0 \times 10^{-5}$ & $\mathrm{sec}$ \\
\hline
\end{tabular}

Table 3: Properties of a copper wire 


\begin{tabular}{lcc}
\hline Entity & Constant Parameters & Frequency Dependent \\
\hline$\varepsilon_{p}$ & $10^{-10}$ & $10^{-10}$ \\
$\varepsilon_{n}$ & $10^{-10}$ & $10^{-10}$ \\
$n$ & 40 & 14 \\
Residual & $5 \times 10^{-16}$ & $4 \times 10^{-19}$ \\
Computation time & $250 \mathrm{sec}$ & $50 \mathrm{sec}$ \\
\hline
\end{tabular}

Table 4: PGD criteria 


\begin{tabular}{lc}
\hline Entity & Parametric Frequency Dependent PGD \\
\hline$\Delta l$ & 0.05 \\
$\varepsilon_{p}$ & $10^{-10}$ \\
$\varepsilon_{n}$ & $10^{-10}$ \\
$n$ & 67 \\
Residual & $1 \times 10^{-7}$ \\
Computation time & $700 \mathrm{sec}$ \\
\hline
\end{tabular}

Table 5: PGD Criteria for parametric solution 